\title{
A high-resolution brain proteome map uncovers the Inter-hemispheric laterality \& Inter-regional protein expression changes
}

\section{Sanjeeva Srivastava ( $\sim$ sanjeeva@iitb.ac.in )}

Indian Institute of Technology Bombay

\section{Deeptarup Biswas}

Indian Institute of Technology Bombay

\section{Sanjyot Shenoy}

Indian Institute of Technology Bombay

Chetanya Chetanya

Indian Institute of Technology Bombay

Arunachalam Athithyan

Indian Institute of Technology Bombay

Mercedes Lachén-Montes

Navarra Institute for Health Research

\section{Susmita Ghosh}

Indian Institute of Technology Bombay

Karina Ausín

Navarra Institute for Health Research

María Zelaya

Navarra Institute for Health Research

Joaquín Fernández-Irigoyen

Navarra Institute for Health Research

\section{Akash Manna}

Medical College Hospital Kolkata

\section{Sudesh Roy}

Indian Institute of Technology Bombay

\section{Abhilash Barpanda}

Indian Institute of Technology Bombay

\section{Graham Ball}

Nottingham Trent University https://orcid.org/0000-0001-5828-7129

\section{Enrique Santamaría}

Complejo Hospitalario de Navarra

\section{Arunansu Talukdar}




\section{Article}

Keywords: Proteomics Investigation, Inter-hemispheric Differences, Synapse Analysis, Protein Markers

Posted Date: December 11th, 2020

DOI: https://doi.org/10.21203/rs.3.rs-88156/v1

License: (c) (1) This work is licensed under a Creative Commons Attribution 4.0 International License. Read Full License 


\section{Abstract}

The human brain has always been a black box full of mysteries. Here we present one of the most comprehensive proteomics investigation of the brain, focusing on inter-hemispheric differences. An extensive mass spectrometry-based analysis of 19 brain regions from both left and right hemispheres measured more than 3300 proteins and 38700 peptides. This high-resolution data provides a comprehensive coverage of experimentally measured (non-hypothetical) proteins across various regions to characterize inter-hemispheric differences. We also tried to understand the brain proteins in terms of synapse analysis. The study has attempted to investigate the expression of neuroanatomical allied region and brain disorder protein markers in 19 region and sub-region of brain. Furthermore, we have developed the most comprehensive Brain Proteome Database, based on our, and publicly available curated data representing more than 9000 proteins (with isoforms) and around 90000 peptides at www.brainprot.org, which can aid in understanding the human brain's complexity.

\section{Introduction}

The human brain is the primary organ of the central nervous system, reflected in its multitude of neurons and glial cells, each of which are home to hundreds of different subtypes chiefly based on their molecular, morphology and connectivity properties ${ }^{1}$. The various synaptic connections between these cell types contribute to the definition of neuro-anatomical sub-divisions in the adult human brain, which is estimated to be around 900 in number and are grouped under two distinct hemispheres. The average weight and overall size of the left and right hemispheres are very similar in healthy adults, but both differ in their functional and anatomical levels ${ }^{2}$. Regarding brain laterality, the left hemisphere specializes in language processing, while the right hemisphere specializes in attention, visuospatial tasks, and many aspects of emotion. Lateralization is accompanied by structural asymmetries that vary with handedness, gender, age, and a variety of genetic, neuropsychiatric, and neurodegenerative conditions ${ }^{3-5}$. The functionality of these hemispheres and different neuroanatomical regions of brain has been revealed in terms of specific regional transcriptional signatures that are regulated in a spatiotemporal manner across mammalian brains ${ }^{6-11}$.

The HBPP under the patronage of the Human Proteome Organization (HUPO) has initiated, established and conducted many proteomics-based studies, which has provided detailed insights of multiple neuroanatomical regions and neuronal compartments (such as neuromelanin granules, mitochondria, nucleus, membranes, axogliasomes, postsynaptic density and myelin) ${ }^{12,13}$. This initiative has created a draft of a human brain proteome database and a sub-cellular reference proteome of a healthy human brain ${ }^{14}$. Proteins functionally regulate most of the biological functions, and the systematic application of neuroproteomics is needed to functionally interpret the information provided by transcriptome-wide approaches ${ }^{1}$. Until now, all proteomic characterizations performed in the human brain have been 
unilateral, leaving a gap in our knowledge of the functional proteomic lateralization of the brain ${ }^{15}$. This study aims to bridge that gap and extend the initiatives of HBPP (The Human Brain Proteome Project) by performing proteome-based investigations of 19 neuro-anatomical regions from both left and right hemisphere Additionally, the findings of inter-region-based analysis have been integrated with different publicly available proteomic datasets of different neuroanatomical regions of human brain. We have also tried to check the status of popular marker proteins of Cerebrospinal Fluid (CSF), Meninges, Spinal Cord, Brain cell and different brain disorders in different neuroanatomical regions of brain. Finally, we have developed an online portal with all the proteins and peptides which could be use by neuroscientists and researchers to accelerate the investigation in inter-hemisphere and neuroanatomical brain regions-based studies. In summary, the widespread implantation and establishment of interhemispheric proteomic innovations will surely promote advancing knowledge about the proteomic portrait of the human brain, leading to novel developments in neurologic differential diagnosis and therapies.

\section{Results}

\section{Interhemispheric brain region-based proteome profiling}

A human brain proteome map was generated by including the proteomic profile of 19 different neuroanatomical regions of both the left and right hemispheres. The samples were run unfractionated, primarily so that our findings could be validated using targeted proteomics strategies in the future (Figure 1). The analysed data of both the left and right hemispheres were later integrated, providing a total of more than 3300 proteins and 38700 peptides with 1\% FDR (Supplementary Fig. 1a, Supplementary Data 1). Human Protein Atlas (HPA), Allen Brain Atlas and literature were referred to group the following regions ${ }^{16,17}$. Amygdala, Hippocampus and Basal Ganglia were taken together under Subcortex. Subregions of Basal Ganglia like Putamen and Caudate Nucleus comes under Straitum whereas Lenticular Extern and Lenticular intern belongs to Lenticular Nucleus (Supplementary Data 1). The protein distribution of the left and right hemisphere in terms of different neuroanatomical regions and subregions has been represented in form of a sunburst chart in Fig. 1b \& Supplementary Data 2. The identified peptides from all 19 regions were plotted based on their length and number of missed cleavages. Most of the identified peptides were less than 15 amino acids long and with no missed cleavages (Supplementary Fig. 1c, Supplementary Data 2).

\section{Chromosome Map of Interhemispheric brain proteome}

A total of 3318 proteins were mapped to their corresponding chromosomes ( 22 Chromosomes, $\mathrm{X}, \mathrm{Y}$, Mitochondrial, Unplaced) using the Nextprot repository as a reference, and represented in the form of a circos plot, to provide better visualization of percentage coverage of chromosomes and chromosomal distribution of the data (Supplementary Fig. 1c, Supplementary Data 2). The brain proteome chromosome 
map was further compared with the total human proteome chromosome map, using bar plots which showed that both of them follows a similar trend (Supplementary Fig. 1d).

\section{Comparative Analysis of Neuroanatomical regions of Human Brain}

The neuroanatomical region-based proteomics data of this study has been taken forward and a heat map was drawn with the region enriched protein intensities (Figure 2a). Few of the protein in the heatmap found to be equally enriched in more than one region of sub-regions of Human brain. A list of 549 common protein were found to be expressed in duplicates of both hemisphere of all 19 regions and subregions of brain (Supplementary Fig. 1a, Supplementary Data 1). Neurofascin (NFASC), Glia maturation factor beta (GMFB), Glial fibrillary acidic protein (GFAP) and Neurochondrin (NCDN) are one of the popular brain proteins in human belongs to the common protein identified in this study has been shown in Figure 2b. Few region-enriched proteins of Basal Ganglia and Cerebral Cortex taken from heatmap and shown in the form of Violin for the significant difference with other regions. The human brain regions like Putamen, Lenticular Intern, Lenticular Extern, and Caudate Nucleus were grouped under Basal Ganglia (BG) whereas Frontal Cortex, Parietal Cortex, Occipital Cortex, and Temporal Cortex were grouped under Cerebral Cortex (CX). The correlation analysis of these anatomically related sub-regions found to have score greater than 0.9 shown in the form of correlation plot in Supplementary Fig. 2a. Hippocalcin (HPCA), Protein phosphatase 1 regulatory inhibitor subunit 1B (PPP1R1B), Protein phosphatase 3 catalytic subunit alpha (PPP3CA) and Translocase of outer mitochondrial membrane 70 (TOMM70) were found to be significantly upregulated in Basal Ganglia whereas ATPase $\mathrm{H}+$ transporting V0 subunit $\mathrm{C}$ (ATP6V0C), PHD finger protein 24 (PHF24), Brain abundant membrane attached signal protein 1 (BASP1) and Synuclein alpha (SNCA) were found to be significantly upregulated in Cerebral Cortex when compared with other regions of the brain (Figure 2C and Supplementary Fig. 2B). A list of 7 proteins were found that were specifically identified in only one region or grouped region like Basal Ganglia and Cerebral Cortex which includes Olfactory marker protein (OMP) in Olfactory Bulb and Ankyrin repeat domain 63 (ANKRD63) in Putamen, Lenticular Intern, Lenticular Extern, and Caudate Nucleus of Basal Ganglia (Figure 2D and Supplementary Data 3).

\section{Collation of proteomic profile of human brain hemispheres}

The hemisphere-based proteomics data were shown in the form of heatmap for Cerebral Cortex, Basal Ganglia and Brainstem (includes Substantia Nigra and PONS) (Figure 3A,B,D and Supplementary Data 3) The proteins in these heatmaps are found to be differentially expressed when left and right hemisphere were analysed. Cerebellar Vermis enriched proteins like Cerebellin 1 precursor (CBLN1), Cerebellin 3 precursor (CBLN3), Purkinje cell protein 2 (PCP2), Neurexin 1 (NRXN1) and Neurexin 3 (NRXN3) which are 
found to be upregulated in Right hemisphere when compared with left hemisphere data (Figure 3C). A list of proteins with differential regulation in terms of left and right hemisphere of Dentate Gyrus of Hippocampus found to be mapped with Generation of Neurones (GO:0048699) with an FDR of 1.74e-09 in STRING. Most of the mapped proteins are found to be upregulated in right hemisphere which has been also confirmed using regression analysis (Figure 3E and Supplementary Data 3).

\section{Synaptic gene ontologies and enrichment analysis of human brain proteome}

A list of 549 common proteins (gene names) were taken forward for Synapse gene ontology in SynGO. 124 unique genes and 208 direct descendants of synapse (G0:0045202) were matched with the input list ( $p$-value: 2.32e-40) under cellular component ontology whereas 88 unique genes and 141 direct descendants of process in the synapse were matched ( $p$-value: 2.06e-24) under biological process ontology. 64 annotated genes which includes Synaptosome associated protein 25 (SNAP25), Synapsin I (SYN1), Synaptojanin 1 (SYNJ1), Synaptotagmin 1 (SYT1) are mapped to presynapse (G0:0098793) however 63 annotated genes which includes Synaptosome associated protein 91 (SNAP91), Profilin 2 (PFN2) are mapped to postsynapse (G0:0098794). The distribution of synaptic genes in terms of both biological process and cellular components were shown in Figure 4A (Supplementary Data 4). The common proteins were also analysed in PANTHER classification system to understand gene ontology based on Molecular Function, Cellular Component and Protein Class shown in Supplementary Fig. 3A, 3B and $3 \mathrm{C}$. Furthermore, the total brain proteins were analysed in NetworkAnalyst using KEGG as background database to understand the synapse pathways under Nervous system (hsa09156). Glutamatergic synapse (hsa04724, p-value: 3.25e-6), GABAergic synapse (hsa04727, p-value: 1.05e-6), Cholinergic synapse (hsa04725, p-value: 0.0013), Dopaminergic synapse (hsa04728, p-value: 3.76e-8) and Serotonergic synapse (hsa 04726, p-value: 0.0077 ) are shown in the form of bipartite network with mapped genes (Figure 4B).

\section{Expression of Neuroanatomical area and Disease markers in Human Brain regions}

The neuroanatomical allied regions like spinal cord, CSF, Meninges, Pituitary and brain cells based protein markers were curated from literatures. Two major types of brain-related disorders, brain tumours and neurodegenerative disorders (NDD) were considered for the disease marker analysis. The popular proteins for each of the alarming diseases were chosen with a $p$-value cut off of 0.05 from Pubpular ${ }^{20}$. Literature-based search and the DisGeNet ${ }^{21}$ search were also used to verify the list. Furthermore, the list of disease-based proteins markers and neuroanatomical allied region markers were taken forward to understand the expression in normal human brain regions shown in Supplementary Fig. 3D (Supplementary Data 4). However, the expression of the large number brain disease based proteome markers in human brain regions are currently ongoing to give a better understanding in future. 


\section{Development of Inter-Hemispheric Brain Proteome Map (IBPM) Portal}

A web-based portal is designed to provide easy access to inter-hemisphere-based human brain proteomics data and region-specific proteomic data that is integrated with publicly available datasets. This information is made publicly available at www.brainprot.org, which includes a search tool, comparing the proteins in terms of the left and right hemisphere, while also fetching proteomics information in an interactive and user-friendly environment.

\section{Discussion}

An increase in the knowledge of brain disorders requires a deep understanding of the human brain. A large number of disease-related studies depend on control brain samples to compare the basal level protein expression, which is always challenging. Different popular databases and repositories like Allen Brain Atlas, Human Protein Atlas have taken vast initiatives to provide a brain reference map in terms of microarray-based gene expression data and transcriptomics data respectively, but a gap still lies in terms of mass spectrometry based comprehensive proteomics data. The study attempts to fill some of the gaps in our knowledge by providing a neuro-anatomical region-based brain proteome map. Different neuroanatomical regions of the brain are known to be functionally different from each other despite being a part of the same organ. The differential expression of genes and proteins, together with the diverse functionality of different regions of the brain itself, makes it the most complex organ of the body. This study has, for the first time demonstrated the differential expression of the proteome in 19 different neuro-anatomical regions and also in terms of brain lateralization. A list of 549 common protein can be considered as brain enriched proteins as these proteins were found in all the 19 regions with maximum confidence, 137 proteins found to be matching with elevated in brain Human Protein Atlas. Our investigation has also found proteins which are enriched in one region or a group of anatomically similar regions of human brain. Hippocalcin (HPCA), a neuron-specific calcium-binding protein found a higher expression in Basal Ganglia and also in Hippocampus. This mRNA differential expression of HPCA has also been found to be overexpressed in Putament and Caudate Nucleus of Basal Ganglia followed by Hippocampus in normal tissues according to GTEx. Protein Phosphatase 1 Regulatory Inhibitor Subunit 1B (PP1R1B) which plays an important role in the stimulation of neurotransmitter has also found to be overexpressed in Basal Ganglia when compared with other regions. PHD Finger Protein 24 (PHF24) and ATPase H+ Transporting V0 Subunit C (ATP6V0C) were found to be overexpressed in Cerebral cortex in this study, which found to be correlate with the Brain Atlas of Human Protein Atlas (HPA). Olfactory marker protein (OMP) plays a crucial role in olfactory signaling and odor discrimination were found to be identified only in the Olfactory Bulb in this study. This particular protein has been reported as a key player in accelerating the maturation of olfactory sensory neuron (OSN) ${ }^{23}$. N-Terminal Xaa-Pro-Lys NMethyltransferase 1 (NTMT1) which was identified in brain stem and substantia nigra whereas Serine And Arginine Rich Splicing Factor 11 (SRSF11) found to be identified only in brain stem. The expression of these two proteins found to be corelate with the RNA expression data of Brain Atlas of Human Protein 
Atlas (HPA) though the overexpression of SRSF11 were shown in Cerebellum followed by Midbrain. NUAK family kinase 1 (NUAK1), identified in Frontal cortex, Parietal cortex and Occipital cortex of cerebral cortex has been known to play important role in cell adhesion, cell proliferation and tumor progression. The GTEx mRNA differential expression of NUAK1 reported an overexpression in Frontal cortex in normal tissues. A recent study in mouse model reveals that Nuak1 could be consider as a novel therapeutic entry point for tauopathies ${ }^{24}$. A basal ganglia specific protein Ankyrin Repeat Domain 63 (ANKRD63) found to be identified in Putamen, Lenticular Nucleus and Caudate Nucleus in our study. A study reveals that prominent expression of ANKRD63 has been found during mouse brain development ${ }^{26}$. Chimerin 2 (CHN2), a GTPase-activating protein was identified only in Cerebellar vermis in this study. The mRNA differential expression data GTEx showed that CHN2 is overexpressed in Cerebellum and Cerebellar Hemisphere. These region-based proteins could be used as a region based anatomical protein marker as most of their overexpression has been supported in this study which was mostly reported in terms of mRNA expression in GTEx and Brain Atlas of HPA (Human Protein Atlas).

This study has also tried to investigate the interhemispheric proteomics expression in 19 different regions of human brain for the very first time and found several differentially expressed proteins. We deciphered its uniqueness by providing measurement of hemisphere-enriched proteins in terms of Basal Ganglia, Cerebral cortex and Brain stem. We have also found Cerebellar vermis enriched proteins like NRXN1, NRXN3, PCP2, CBLN1 and CBLN3 which has also been shown with high expression in RNA data of Cerebellum in HPA. These proteins play important role in synaptic signal transmission and found to be upregulated in the right hemisphere in our study $26,27,28$. Our proteome level investigation has also tried to understand the biological pathways like adult hippocampal neurogenesis-based proteins. Neurogenesis, a process of generation of new neurones which are important for emotion, depression and cognition ${ }^{29,30}$. Hippocampus especially dentate gyrus (DG) play important role in adult neurogenesis and behavioural discriminations ${ }^{31,32}$. We have tried to map the dentate gyrus proteome with generation of neurones (GO:0048699), a biological process which results in 18 differentially regulated protein based on left and right hemisphere. 17 proteins which includes Growth Associated Protein-43 (GAP43), Synculin-1 (SYN1), synaptosomal-associated protein 25 (SNAP-25) are found to be upregulated in right hemisphere when compared with left hemisphere. GAP43 plays an important role in neurogenesis whose failure in expression leads into apoptosis of neurones ${ }^{33}$. SYN1 and SNAP2 5 belongs to hippocampal synaptoproteome which regulates neurotransmission ${ }^{34}$. The overexpression of these dentate gyrus proteins in neurogenesis directs towards the active role of right hemisphere but the result needs validation in large cohort of sample.

A large number of studies that are dependent on human control brain regions can refer to this proteome map to get an overview of region based proteomic expression. Furthermore, this study has also included different repositories and published datasets to make it the most comprehensive compendium of human brain proteins. With the availability of the Human Genome, neuroanatomical region-based 
transcriptomics data, our proteomic data corroborates the assets and would serve as a reference map for different neurobiologists and brain disorder studies. This study of a neuroanatomical region-based brain lateralization prototyping might help to accelerate the understanding of the complexity of the brain by researchers and clinicians working worldwide. In light of the detection of lateralized protein expression in post-mortem adult brain data, the future study of embryonic, fetal, and developmental stages in combination with single-cell proteomics will allow obtaining a more comprehensive protein atlas of the human brain.

\section{References}

1. Kitchen, R. R., Rozowsky, J. S., Gerstein, M. B. \& Nairn, A. C. Decoding neuroproteomics: integrating the genome, translatome and functional anatomy. Nat. Neurosci. 17, 1491-1499 (2014).

2. Hawrylycz, M. J. et al. An anatomically comprehensive atlas of the adult human brain transcriptome. Nature 489, 391-399 (2012).

3. Sun, T. \& Walsh, C. A. Molecular approaches to brain asymmetry and handedness. Nat. Rev. Neurosci. 7, 655-662 (2006).

4. Caeyenberghs, K. \& Leemans, A. Hemispheric lateralization of topological organization in structural brain networks. Hum. Brain Mapp. 35, 4944-4957 (2014).

5. Gainotti, G. Laterality effects in normal subjects' recognition of familiar faces, voices and names. Perceptual and representational components. Neuropsychologia 51, 1151-1160 (2013).

6. Kang, H. J. et al. Spatio-temporal transcriptome of the human brain. Nature 478, 483-489 (2011).

7. Miller, J. A. et al. Transcriptional landscape of the prenatal human brain. Nature 508, 199-206 (2014).

8. Lein, E. S. et al. Genome-wide atlas of gene expression in the adult mouse brain. Nature 445, 168176 (2007).

9. Morris, J. A. et al. Divergent and nonuniform gene expression patterns in mouse brain. Proc. Natl. Acad. Sci. 107, 19049 (2010).

10. Bakken, T. E. et al. A comprehensive transcriptional map of primate brain development. Nature 535, 367-375 (2016).

11. Xu, C. et al. Human-specific features of spatial gene expression and regulation in eight brain regions. Genome Res. 28, 1097-1110 (2018).

12. Reidegeld, K. A. et al. The power of cooperative investigation: Summary and comparison of the HUPO Brain Proteome Project pilot study results. PROTEOMICS 6, 4997-5014 (2006).

13. Stephan, C. et al. Automated reprocessing pipeline for searching heterogeneous mass spectrometric data of the HUPO Brain Proteome Project pilot phase. PROTEOMICS 6, 5015-5029 (2006).

14. Mueller, M. et al. Functional annotation of proteins identified in human brain during the HUPO Brain Proteome Project pilot study. PROTEOMICS 6, 5059-5075 (2006). 
15. Fernandez-Irigoyen, J. et al. Toward defining the anatomo-proteomic puzzle of the human brain: An integrative analysis. PROTEOMICS - Clin. Appl. 9, 796-807 (2015).

16. Sunkin, S. M. et al. Allen Brain Atlas: an integrated spatio-temporal portal for exploring the central nervous system. Nucleic Acids Res. 41, D996-D1008 (2012).

17. Uhlen, M. et al. Towards a knowledge-based Human Protein Atlas. Nat. Biotechnol. 28, 1248-1250 (2010).

18. Pang, Z., Zuo, J. \& Morgan, J. I. Cbln3, a Novel Member of the Precerebellin Family that Binds Specifically to Cbln1. J. Neurosci. 20,6333 (2000).

19. Caporaso, G. L. et al. Drugs of abuse modulate the phosphorylation of ARPP-21, a cyclic AMPregulated phosphoprotein enriched in the basal ganglia. Neuropharmacology 39, 1637-1644 (2000).

20. Lam, M. P. Y. et al. Data-Driven Approach To Determine Popular Proteins for Targeted Proteomics Translation of Six Organ Systems. J. Proteome Res. 15, 4126-4134 (2016).

21. Piñero, J. et al. The DisGeNET knowledge platform for disease genomics: 2019 update. Nucleic Acids Res. 48, D845-D855 (2019).

22. Koopmans, F. et al. SynGO: An Evidence-Based, Expert-Curated Knowledge Base for the Synapse. Neuron 103, 217-234.e4 (2019).

23. Albeanu, D.F., Provost, A.C., Agarwal, P., Soucy, E.R., Zak, J.D. and Murthy, V.N., 2018. Olfactory marker protein (OMP) regulates formation and refinement of the olfactory glomerular map. Nature communications, 9(1), pp.1-12.

24. Lasagna-Reeves, C.A., de Haro, M., Hao, S., Park, J., Rousseaux, M.W., Al-Ramahi, I., Jafar-Nejad, P., Vilanova-Velez, L., See, L., De Maio, A. and Nitschke, L., 2016. Reduction of Nuak1 decreases tau and reverses phenotypes in a tauopathy mouse model. Neuron, 92(2), pp.407-418.

25. Tissir, F., Bar, I., Goffinet, A.M. and Lambert De Rouvroit, C., 2002. Expression of the ankyrin repeat domain 6 gene (Ankrd6) during mouse brain development. Developmental dynamics: an official publication of the American Association of Anatomists, 224(4), pp.465-469.

26. Wei, P., Pattarini, R., Rong, Y., Guo, H., Bansal, P.K., Kusnoor, S.V., Deutch, A.Y., Parris, J. and Morgan, J.I., 2012. The Cbln family of proteins interact with multiple signaling pathways. Journal of neurochemistry, 121(5), pp.717-729.

27. Guan, J., Luo, Y. and Denker, B.M., 2005. Purkinje cell protein-2 (Pcp2) stimulates differentiation in PC12 cells by GßY-mediated activation of Ras and p38 MAPK. Biochemical Journal, 392(2), pp.389397.

28. Uemura, T., Lee, S.J., Yasumura, M., Takeuchi, T., Yoshida, T., Ra, M., Taguchi, R., Sakimura, K. and Mishina, M., 2010. Trans-synaptic interaction of GluRס2 and Neurexin through Cbln1 mediates synapse formation in the cerebellum. Cell, 141(6), pp.1068-1079.

29. Sahay, A. and Hen, R., 2007. Adult hippocampal neurogenesis in depression. Nature neuroscience, 10(9), pp.1110-1115. 
30. Baptista, P. and Andrade, J.P., 2018. Adult hippocampal neurogenesis: regulation and possible functional and clinical correlates. Frontiers in neuroanatomy, 12, p.44.

31. Amaral, D.G., Scharfman, H.E. and Lavenex, P., 2007. The dentate gyrus: fundamental neuroanatomical organization (dentate gyrus for dummies). Progress in brain research, 163, pp.3790.

32. Christian, K.M., Ming, G.L. and Song, H., 2020. Adult neurogenesis and the dentate gyrus: Predicting function from form. Behavioural brain research, 379, p.112346.

33. Mani, S., Shen, Y., Schaefer, J. and Meiri, K.F., 2001. Failure to express GAP-43 during neurogenesis affects cell cycle regulation and differentiation of neural precursors and stimulates apoptosis of neurons. Molecular and Cellular Neuroscience, 17(1), pp.54-66.

34. VanGuilder, H.D., Yan, H., Farley, J.A., Sonntag, W.E. and Freeman, W.M., 2010. Aging alters the expression of neurotransmission-regulating proteins in the hippocampal synaptoproteome. Journal of neurochemistry, 113(6), pp.1577-1588.

\section{Methods}

\section{Histopathological Study}

Informed written consent was obtained from relatives of a 45-year-old male to perform clinical autopsy. The use of brain sample was carried out according to the current guidelines of Spanish legislation (Real Decreto 2011/1716) and the Declaration of Helsinki and all assessments. The autopsy confirmed peritonitis with intestinal perforation, infiltrated by ring cell adenocarcinoma and disseminated metastasis. After the brain removal from the skull, forty-two fresh samples (21 brain areas derived from both cerebral hemispheres) were extracted in parallel without delay, immediately frozen and stored at -80 ${ }^{\circ} \mathrm{C}$ for proteomic analysis. The areas assessed in this study were: Olfactory bulb (OB), Substantia nigra $(\mathrm{SN})$, Spinal cord (SpC), Brainstem (BS), Pons (Prot/Pons), Cerebellar vermis (CV), Dentate gyrus (DG), Cingulum (CING), Motor cortex (MCx), Putamen (PUT), Caudate nucleus (CN), Lenticular extern (LE), lenticular intern (LI), Frontal cortex (FC), Temporal cortex (TC), Thalamus (TH), Hippocampus (HIP), Amygdala (AMY), Parietal cortex (PC), Occipital cortex (OC), and Frontal white matter (FWM). The remaining brain areas were fixed with $4 \%$ buffered formalin for 4 weeks and some other selected regions were embedded in paraffin and stained with haematoxylin and eosin following the BrainNet Europe guidelines $^{35}$. Protein expression of Tau, $\beta$-amyloid, TDP-43, $\alpha$-synuclein, ubiquitin, and $a-\beta$ crystalline was analysed by immunohistochemistry using specific antibodies across different brain regions. The microscopic study demonstrated minor neuronal hypoxic changes in the temporal and hippocampal area. No neuropathological signs of neurodegenerative disorders were found on pathological examination of the subject.

\section{Sample preparation for LFQ analysis}


Protein extraction was performed as previously described ${ }^{36}$. Brain samples were homogenized in lysis buffer containing $7 \mathrm{M}$ urea, $2 \mathrm{M}$ thiourea and $50 \mathrm{mM}$ DTT supplemented with protease and phosphatase inhibitors. Then, samples were spun down at $100,000 \times \mathrm{g}$ for one hour at $15^{\circ} \mathrm{C}$. After protein precipitation, protein concentration in the supernatant was measured with the Bradford assay kit (Biorad). Protein enzymatic cleavage was carried out with trypsin (Promega; $1: 20, \mathrm{w} / \mathrm{w}$ ) at $37^{\circ} \mathrm{C}$ for $16 \mathrm{~h}$. Peptides were subjected to desalting (C18-sep packs column, Merck Milipore) and vacuum dried. Reconstitution of peptides was undertaken using $0.1 \%$ Formic acid, and peptide quantification was performed using Scopes method ${ }^{37}$.

\section{Liquid chromatography tandem mass-spectrometry (LC-MS/MS)}

$1 \mu \mathrm{g}$ of digested and desalted peptides were loaded onto the column at $5 \mu \mathrm{l} / \mathrm{min}$ flow rate. Peptides were resolved on an analytical column at a flow rate of $300 \mathrm{nl} / \mathrm{min}$ over $180 \mathrm{~min}$ gradient in solvent B (80\% $\mathrm{ACN}$ in $0.1 \% \mathrm{FA}$ ). Mass spectrometric acquisition was performed in the DDA (Data Dependent Acquisition) mode in full scan range of 375-1700 m/z with Orbitrap fusion mass analyser at a mass resolution of 60,000 in Mass Spectrometry Facility at IIT Bombay (MASSFIITB). The mass window was set to be 10 ppm with a dynamic exclusion duration of 40s. All MS/MS spectra were acquired by HCD, i.e. High energy Collision Dissociation method of fragmentation. Each sample was run in duplicates in a randomized manner to prevent a run-to-run bias and remove prevent batch variation.

\section{LFQ data analysis in MaxQuant}

The raw datasets were processed with MaxQuant (v1.6.5.0) against UniProt Human Proteome Database (Proteome ID: UP000005640) and searched with the built-in Andromeda Search Engine of MaxQuant ${ }^{38}$. Raw files were processed within Label-Free-Quantification (LFQ) parameters setting label-type as "standard" with a multiplicity of 1 . The Orbitrap was set to Orbitrap Fusion mode. Trypsin was used for digestion with a maximum of 2 missed cleavages. Carbamidomethylation of Cysteine (+57.021464 Da) was set as the fixed modification, whereas oxidation of Methionine (+15.994915 Da) was set as the variable modification. The False-Discovery-Rate (FDR) was set to 1\% for the protein and peptide levels to ensure high reliability of the protein detection/identification. The minimum length for a peptide was set to 7AA. Decoy mode was set to "randomize", and the type of identified peptides was set to "unique+razor".

\section{Data quality check and analysis}

The analysis of 21 samples showed that the left hemisphere of Motor Cortex (MCx) sample were not giving appropriate number of proteins. The sample was re-run for confirmation and $\mathrm{MCx}$ sample was 
excluded from the study. The max quant analysis was performed with 20 samples and the data was taken forward for further analysis.

Statistical and bioinformatics analysis of spectra and proteomics data was performed using Metaboanalyst ${ }^{39}$, Microsoft Excel, Python, and R statistical software. No missing value imputation has been done. The hierarchical clustering was performed using z-score transformed LFQ intensities and was further clustered using Euclidean as a distance in Metaboanalyst ${ }^{39}$ and Hierarchical Clustering Explorer $^{40}$ (Version 3.5). PANTHER ${ }^{41}$, WebGestalt ${ }^{42}$ and SynGO were used to perform some pathway enrichment and synapse biology-based on Gene Ontology (GO). STRING and NetworkAnalyst were used for protein-protein interaction and enrichment analysis.

\section{Regression/Residual Analysis of inter-hemispheric data}

A simple bivariate linear regression analysis, used to identify significantly over or under-expressed proteins associated with a particular sample was performed. In this approach, the protein expression levels were regressed for pairs of individuals. For example, the expression levels for a replicate of AMY were put on the $\mathrm{Y}$-axis, and the expression levels for another non-AMY sample were put on the $\mathrm{X}$-axis. A regression analysis was conducted in MS Excel and standard residual values were calculated for each protein for the regression. This approach was repeated for all replicates in a pair-wise fashion, regressing all of the sample profiles in the region against all sample profiles out of the region. The mean residual value was calculated for a given region. The advantage of this approach is that it combines the power of a volcano plot with the ability to examine replicate-to-replicate variation and region-to-region variation. For each regression, the distribution of residuals and the correlation coefficients were examined. Raw regression results are presented in the supplementary data. This approach was not only used to look at the region-specific proteins but also used to examine the Left versus Right expression for each region. In this way, the Left v/s Right differential expression could be examined across all regions.

\section{Data mining and data extraction for comparative analysis}

Proteomics study with keywords related to the human brain and different neuro-anatomical regions of the human brain, according to the Allen Brain Atlas Classification table were searched in Proteome Exchange and PRIDE repository. All the studies available in the repository up to 10.02 .2020 with the following filter criteria - Species: Human (Homo sapiens) and Method: LFQ were downloaded. The manuscript of the papers and sample metadata files were thoroughly studied to understand the experimental design and which raw files belong to the healthy subjects. In most cases, authors were contacted to verify the raw files. Additionally, in-house generated mass spectrometry-based data of few other regions from our previous study were also taken. All these curated raw mass spectrometry files were taken forward for MaxQuant data analysis with Human Proteome Database (Isoform). Further, brain-related databases and 
repositories were also considered, which included Brain Atlas of Human Protein Atlas (HPA), CSF Proteome Resource (CSF-PR) ${ }^{43}$, and Harmonizome ${ }^{44}$.

\section{Inter-hemispheric brain proteome map (IBPM) portal}

The IBPM portal under www.brainprot.org is designed on the Django framework with robust features for visualization of data in the study and it will act as an interface for the researchers in the field of brain proteomics for data integration and region-specific information. The portal also fetches general information like the chromosome localization, function, and peptide information about the protein from various existing repositories. The portal is designed with scalability at its core; the database in the portal is highly dynamic and can be extended to incorporate public databases.

\section{Data availability}

All MS raw files could be accessed from Proteome-Xchange using the identifier PXD019936.

\section{References}

35. Bell, J. E. et al. Management of a twenty-first century brain bank: experience in the BrainNet Europe consortium. Acta Neuropathologica 115, 497-507 (2008).

36. Ferrer, I. et al. Familial globular glial tauopathy linked to MAPT mutations: molecular neuropathology and seeding capacity of a prototypical mixed neuronal and glial tauopathy. Acta Neuropathologica 139, 735-771 (2020).

37. Scopes, R. K. Measurement of protein by spectrophotometry at $205 \mathrm{~nm}$. Analytical Biochemistry 59, 277-282 (1974).

38. Tyanova, S., Temu, T. \& Cox, J. The MaxQuant computational platform for mass spectrometry-based shotgun proteomics. Nature Protocols 11, 2301-2319 (2016).

39. Chong, J. et al. MetaboAnalyst 4.0: towards more transparent and integrative metabolomics analysis. Nucleic Acids Research 46, W486-W494 (2018).

40. Seo, J. \& Shneiderman, B. Interactively exploring hierarchical clustering results [gene identification]. Computer 35, 80-86 (2002).

41. Mi, H., Muruganujan, A., Ebert, D., Huang, X. \& Thomas, P. D. PANTHER version 14: more genomes, a new PANTHER GO-slim and improvements in enrichment analysis tools. Nucleic Acids Research 47, D419-D426 (2018).

42. Liao, Y., Wang, J., Jaehnig, E. J., Shi, Z. \& Zhang, B. WebGestalt 2019: gene set analysis toolkit with revamped Uls and APIs. Nucleic Acids Research 47, W199-W205 (2019).

43. Guldbrandsen, A. et al. CSF-PR 2.0: An Interactive Literature Guide to Quantitative Cerebrospinal Fluid Mass Spectrometry Data from Neurodegenerative Disorders. Molecular \&amp;amp; Cellular Proteomics 16, 300 (2017). 
44. Rouillard, A. D. et al. The harmonizome: a collection of processed datasets gathered to serve and mine knowledge about genes and proteins. Database 2016, (2016)

\section{Figures}

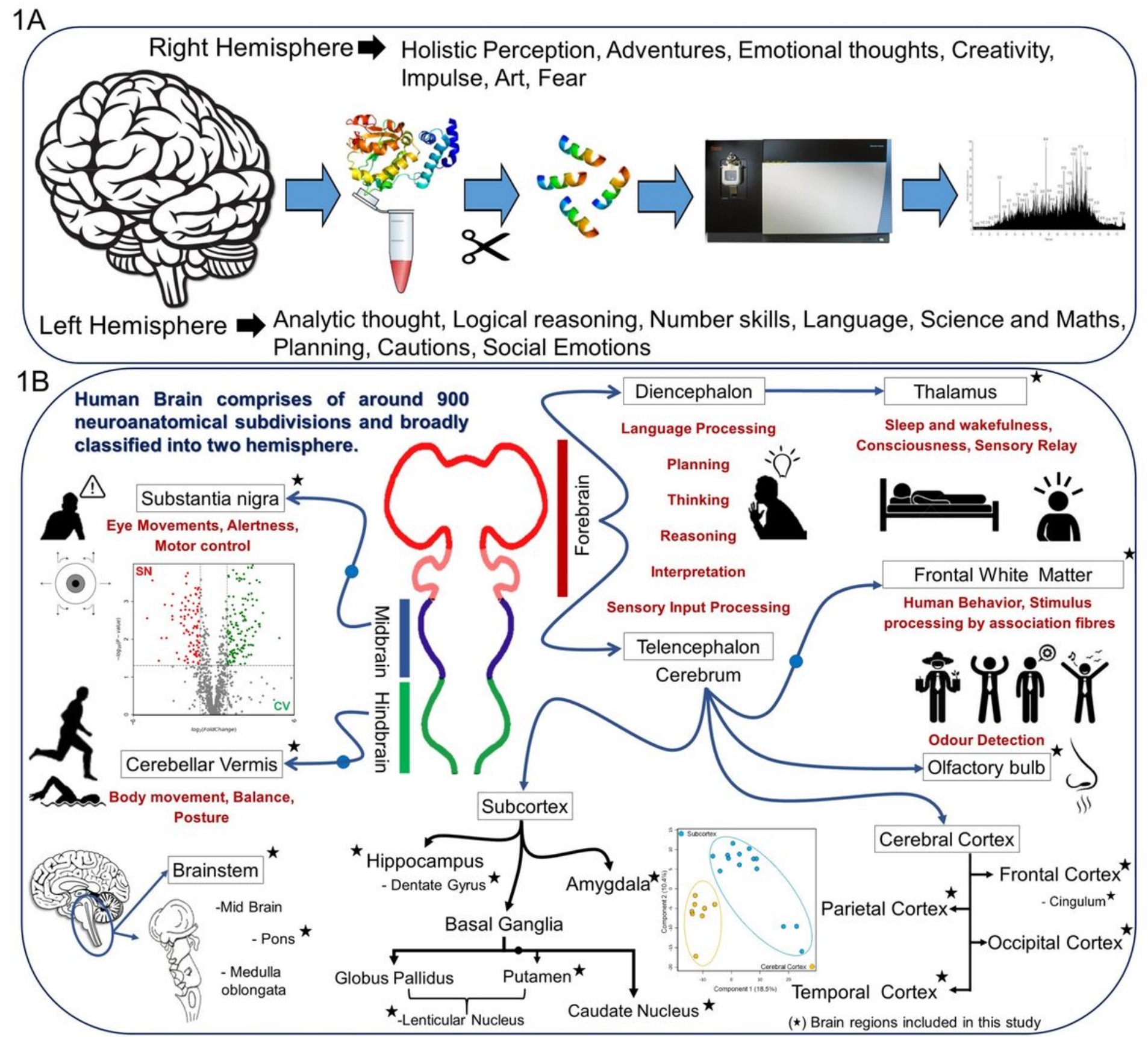

Figure 1 
Anatomical classification map of Human Brain; Figure 1A demonstrates the experimental plan of human brain proteome map and Figure 1B shows the anatomical classification of human brain where 19 region and sub-regions of brain which has been considered in this study has been highlighted with asterisk $\left(^{*}\right)$. The sub-cortex which includes Amygdala, Hippocampus, Dentate Gyrus, Putamen, Lenticular Nucleus and Caudate Nucleus founds to be perturbed in a PCA with cerebral cortex which includes Parietal cortex, Frontal cortex, Occipital cortex and Temporal cortex. A volcano plot between Substantia nigra and Cerebellar vermis shows a large number of proteins found to be differentially regulated.

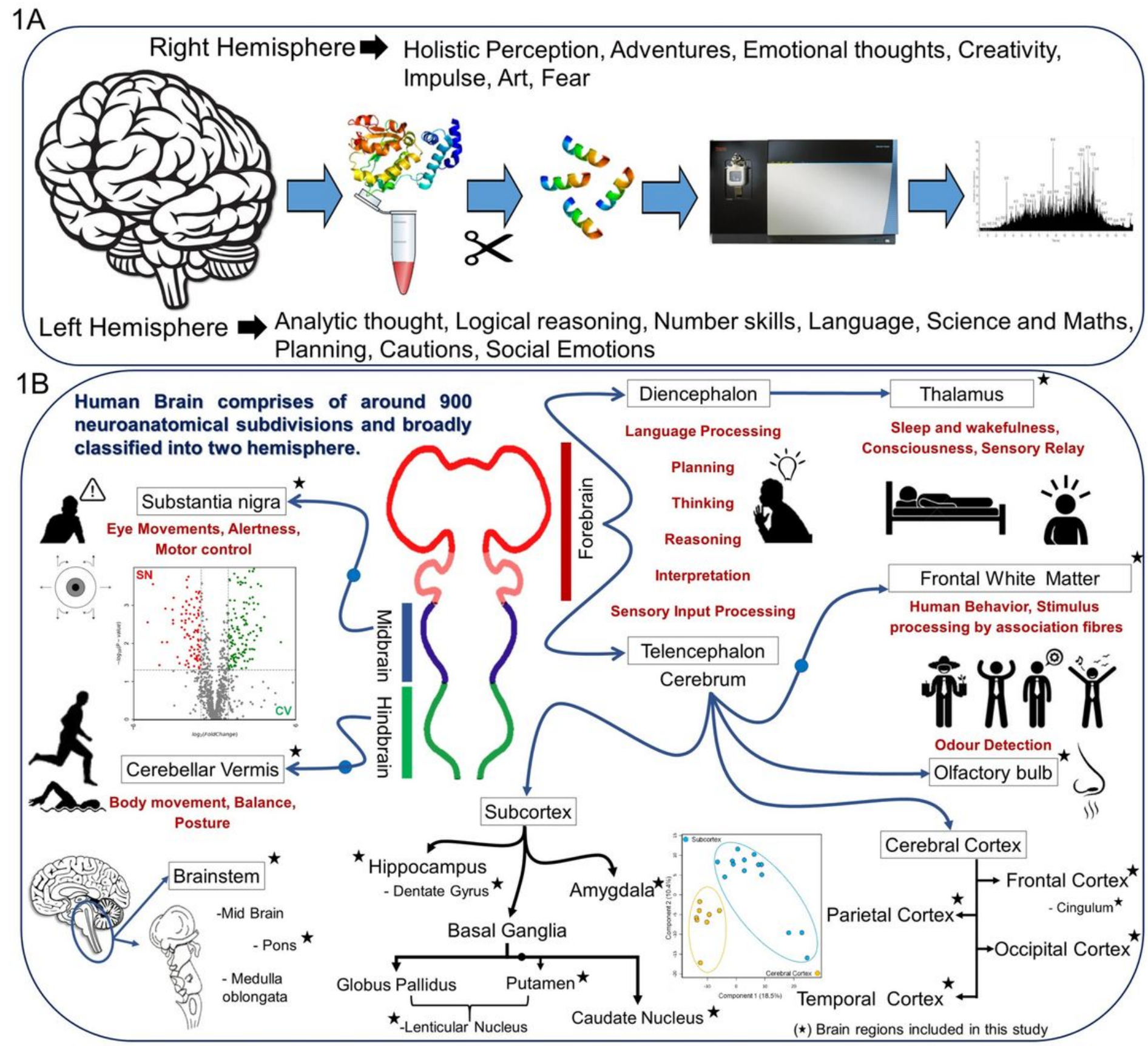


Anatomical classification map of Human Brain; Figure 1A demonstrates the experimental plan of human brain proteome map and Figure 1B shows the anatomical classification of human brain where 19 region and sub-regions of brain which has been considered in this study has been highlighted with asterisk $\left(^{\star}\right)$. The sub-cortex which includes Amygdala, Hippocampus, Dentate Gyrus, Putamen, Lenticular Nucleus and Caudate Nucleus founds to be perturbed in a PCA with cerebral cortex which includes Parietal cortex, Frontal cortex, Occipital cortex and Temporal cortex. A volcano plot between Substantia nigra and Cerebellar vermis shows a large number of proteins found to be differentially regulated.

$2 \mathrm{~A}$
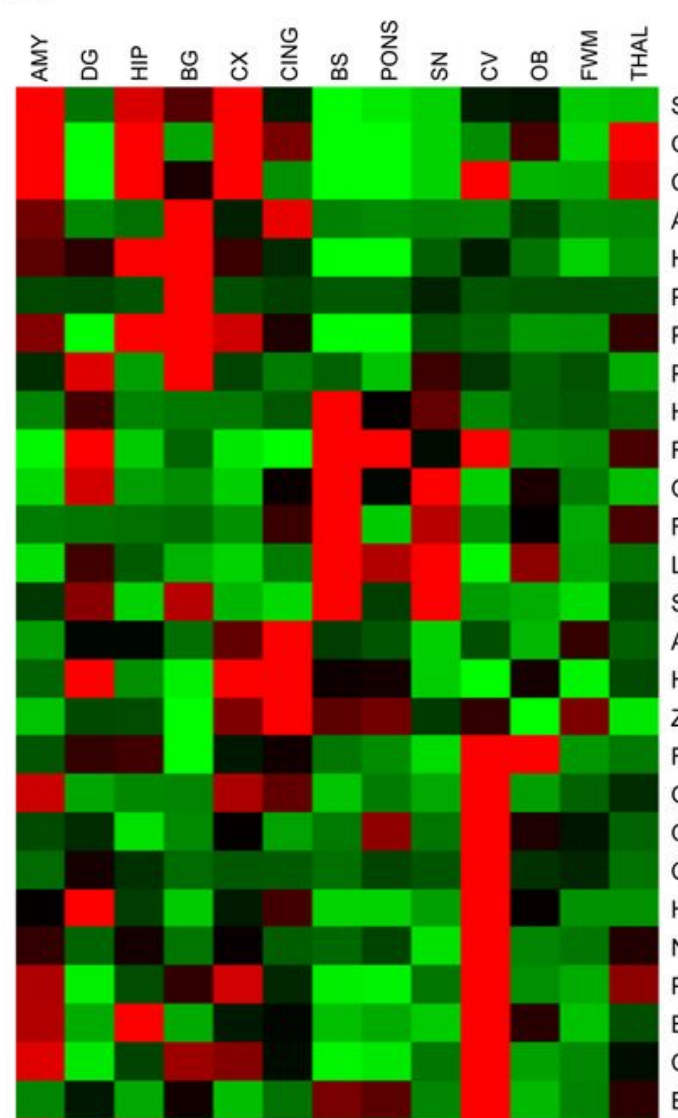

SLC8A2

WDR47
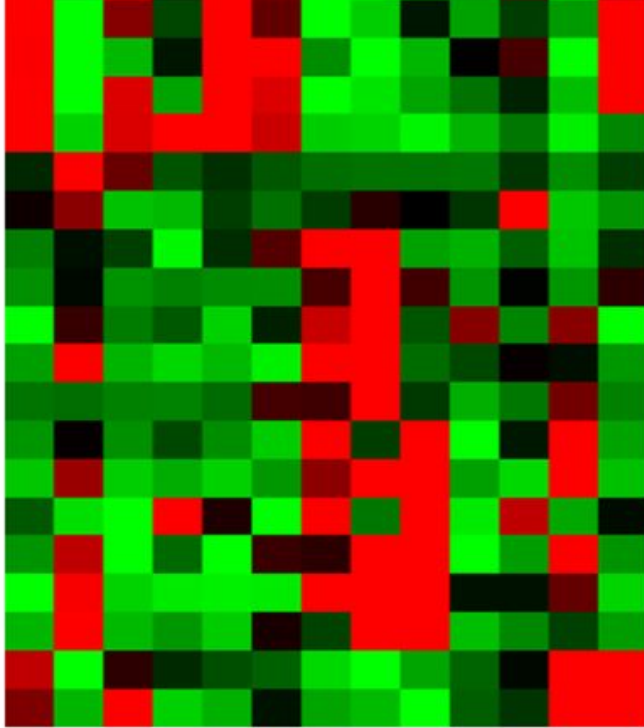

GAP43

ATP6VOC

BASP1

SYNPO

PRKCG

PLIN4

RPS2

CRABP 1

RANGAP1

PLCD1

AHNAK2

FGF1

NEFH

SYPL1

CPPED1

TUBB2B

ANLN

ACTBL2

XRN2
2B

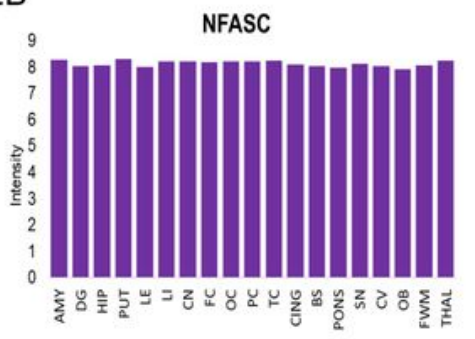

GFAP

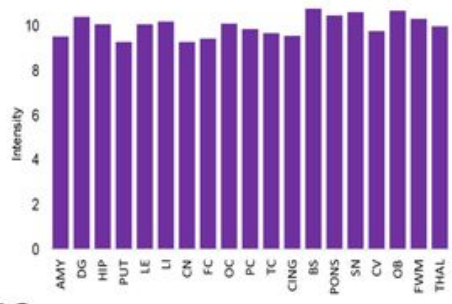

$2 \mathrm{C}$
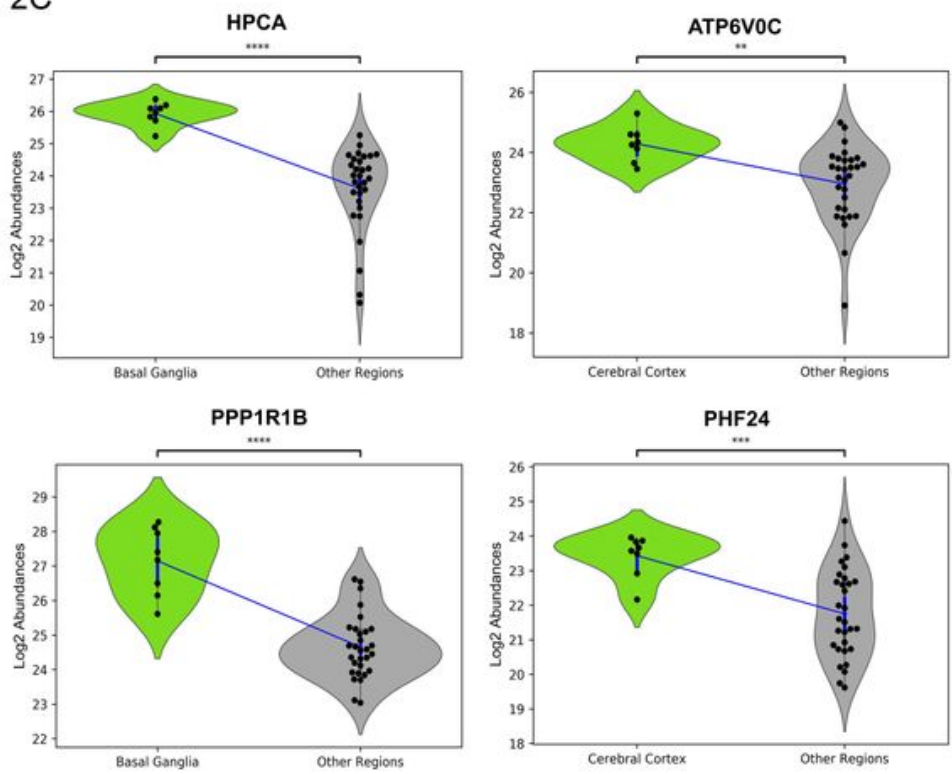

2D
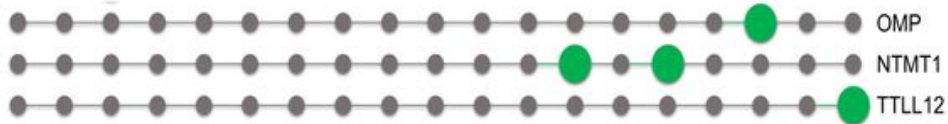

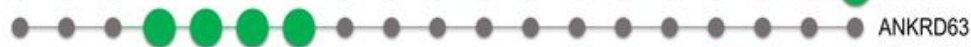
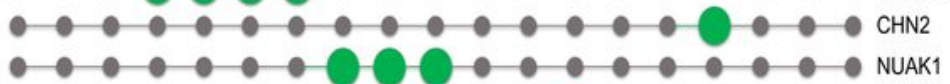

-

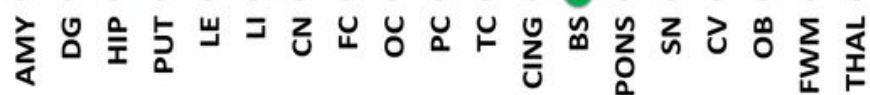




\section{Figure 2}

Inter-region proteomic profiling of Human Brain; Figure 2A depicts a heatmap with a list of protein that found to be enriched in different regions of human brain. Figure 2B represents four common proteins which include Neurofascin (NFASC), Glia maturation factor beta (GMFB), Glial fibrillary acidic protein (GFAP) and Neurochondrin (NCDN) found in all the 19 regions of human brain shown in the form of bar plot. Figure $2 \mathrm{C}$ depicts the proteins like Neuron-specific calcium-binding protein hippocalcin (HPCA), Protein phosphatase 1 regulatory subunit 1B (PPP1R1B), V-type proton ATPase $16 \mathrm{kDa}$ proteolipid subunit (ATP6V0C) and PHD finger protein 24 (PHF24) in the form of violin plot. (ns: $5.00 \mathrm{e}-02<\mathrm{p}<=$ 1.00e+00; : 1.00e-02 < p <= 5.00e-02; *: 1.00e-03<p<=1.00e-02; *: 1.00e-04 < p <= 1.00e-03; **: $p<=$ $1.00 \mathrm{e}-04)$ Figure $2 \mathrm{D}$ represents 7 protein specific to one region of human brain. 

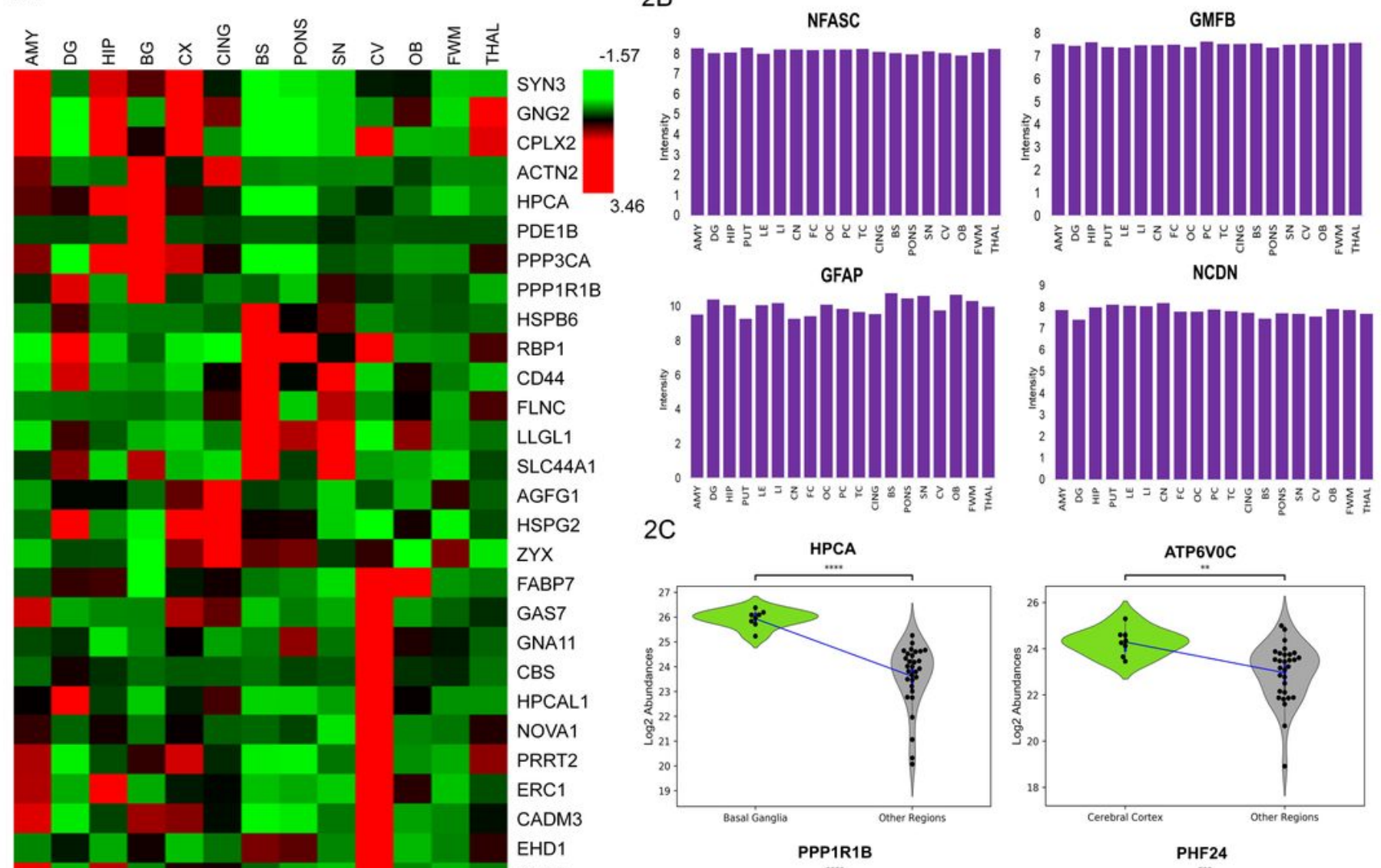

PSD3

SLC8A2 WDR47 GAP43 ATP6VOC BASP1 SYNPO PRKCG PLIN4 RPS2 CRABP1
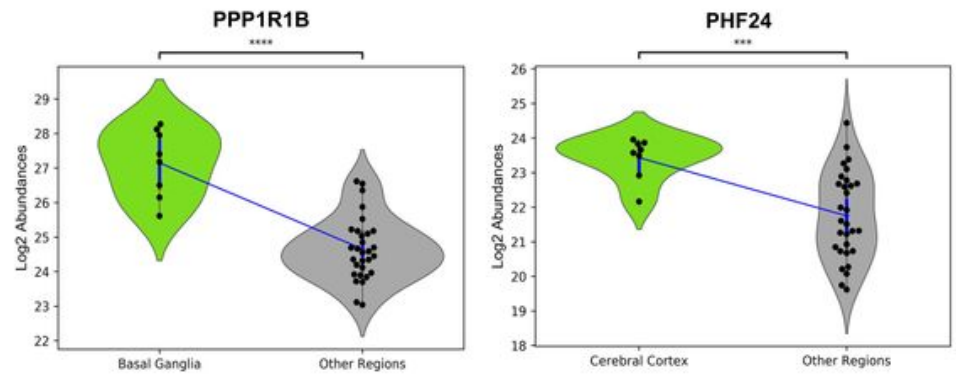

2D

RANGAP1

PLCD1

AHNAK2

FGF1

NEFH

SYPL1

CPPED1

TUBB2B

ANLN

ACTBL2 XRN2

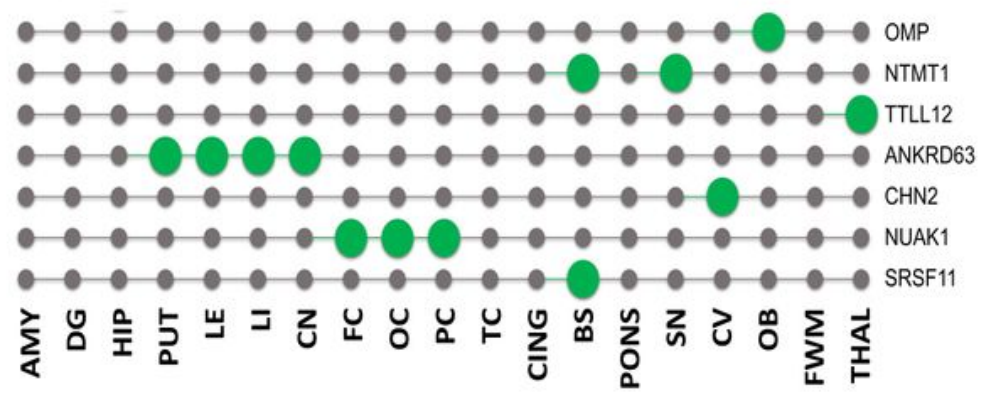

Figure 2

Inter-region proteomic profiling of Human Brain; Figure 2A depicts a heatmap with a list of protein that found to be enriched in different regions of human brain. Figure 2B represents four common proteins which include Neurofascin (NFASC), Glia maturation factor beta (GMFB), Glial fibrillary acidic protein (GFAP) and Neurochondrin (NCDN) found in all the 19 regions of human brain shown in the form of bar plot. Figure 2C depicts the proteins like Neuron-specific calcium-binding protein hippocalcin (HPCA), 
Protein phosphatase 1 regulatory subunit 1B (PPP1R1B), V-type proton ATPase $16 \mathrm{kDa}$ proteolipid subunit (ATP6VOC) and PHD finger protein 24 (PHF24) in the form of violin plot. (ns: $5.00 \mathrm{e}-02<\mathrm{p}<=$ 1.00e+00; : 1.00e-02 < p <= 5.00e-02; *: 1.00e-03<p<= 1.00e-02; *: 1.00e-04 < p <= 1.00e-03; **: $p<=$ $1.00 \mathrm{e}-04)$ Figure $2 \mathrm{D}$ represents 7 protein specific to one region of human brain.
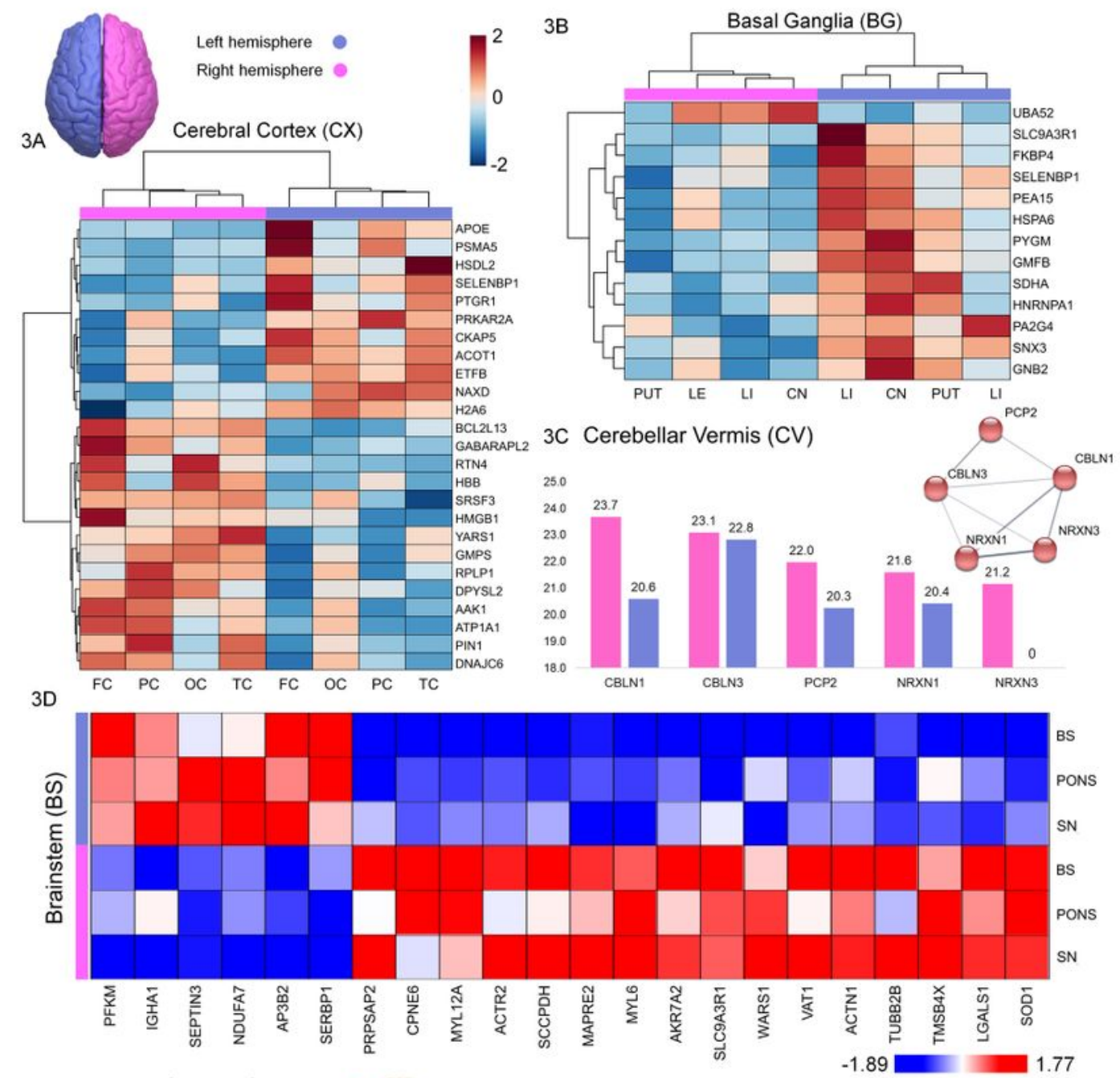

3E Dentate Gyrus (DG)
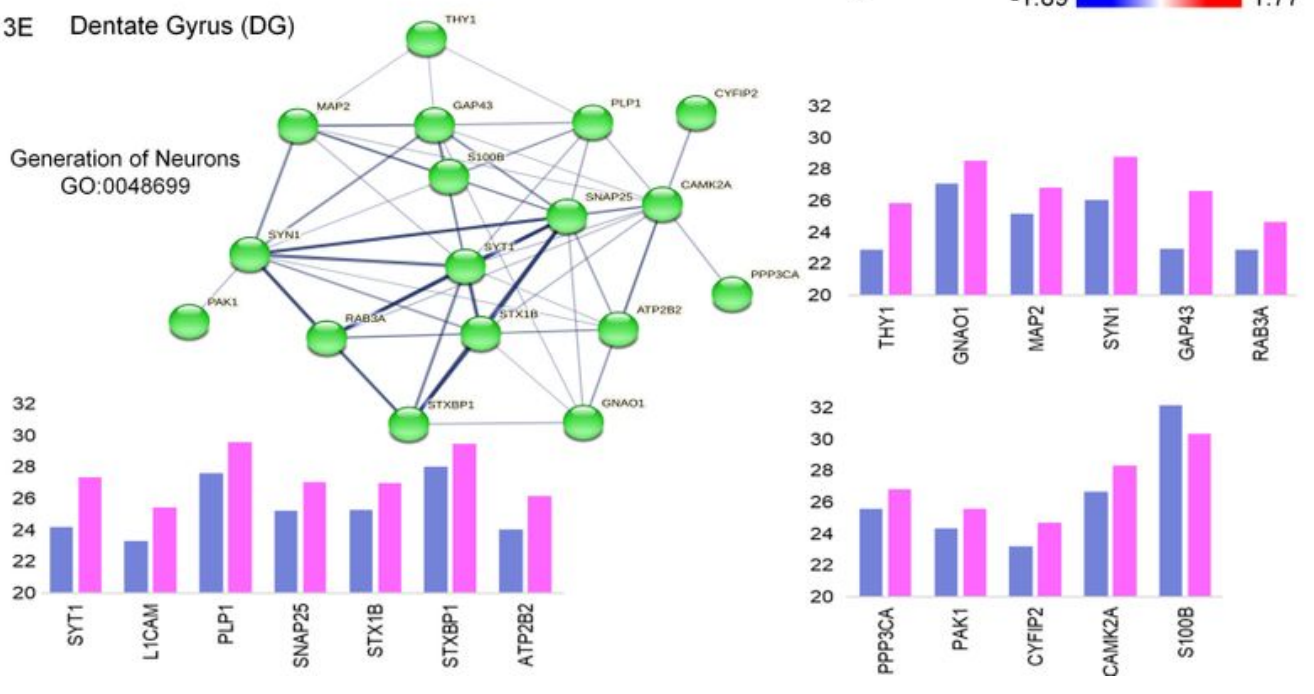

Figure 3 
Inter-hemispheric proteomic profile of human brain; Figure 3A illustrates the list of significant proteins which segregates Left hemisphere and Right hemisphere of Cerebral Cortex (Frontal Cortex, Occipital Cortex, Temporal and Parietal Cortex). Figure 3B depicts the list of proteins which segregates Left hemisphere and Right hemisphere of Basal Ganglia (Putamen, Lenticular nuclei and Caudate Nucleus). Figure $3 \mathrm{C}$ portrays the expression of few Cerebellar vermis enriched protein in terms of both the hemisphere and also the protein-protein interaction map of CBLN3, CBLN1, PCP2, NRXN1 and NRXN2. Figure $3 \mathrm{E}$ depicts the perturbation of interhemispheric regions of Brainstem, Pons and Substantia nigra. Figure $3 \mathrm{~F}$ shows the protein-protein interaction of a biological process, Generation of Neurons (G0:0048699) with 18 Dentate Gyrus protein (17 Upregulated in Right hemisphere and 1 Downregulated in Left hemisphere). 

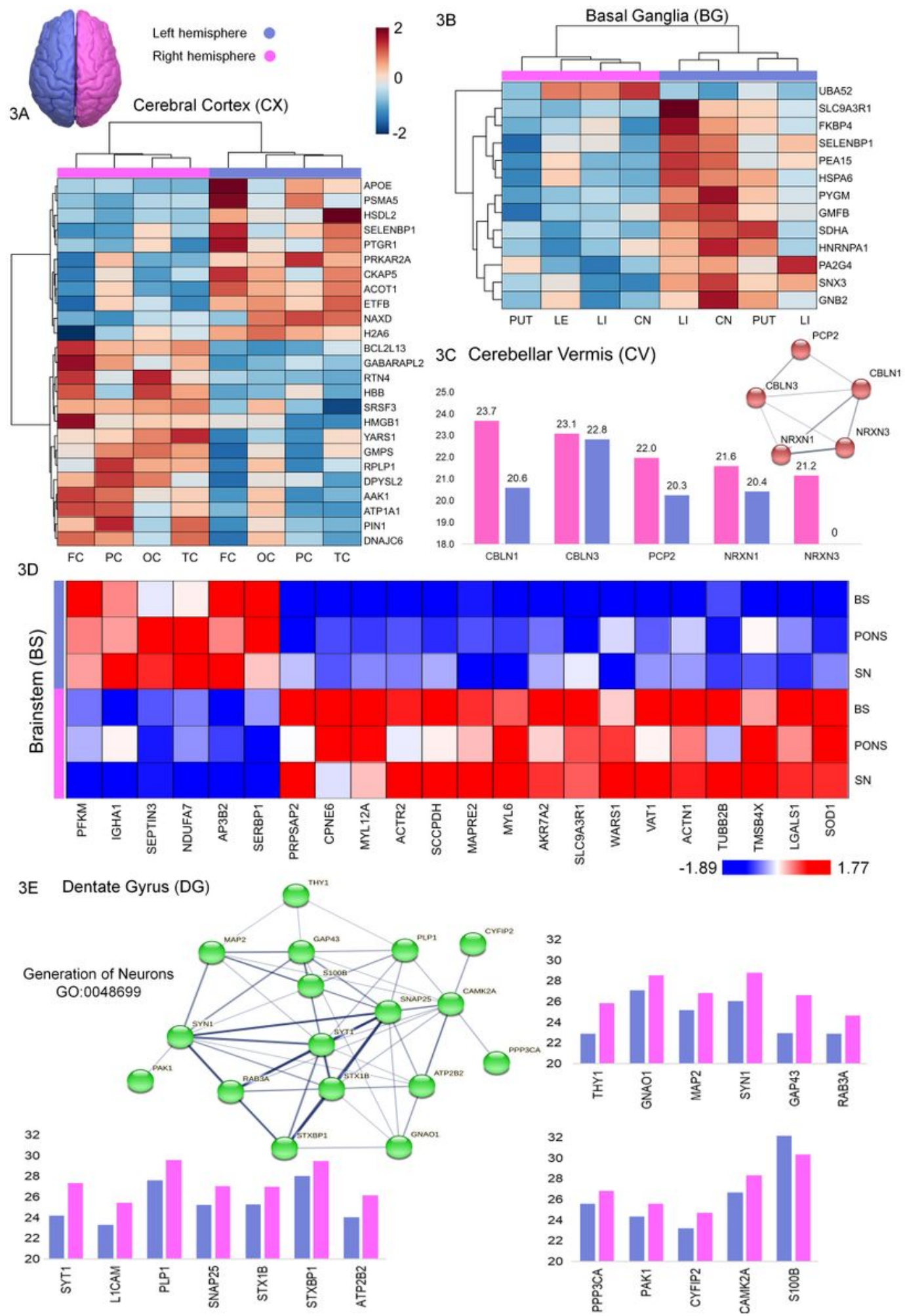

\section{Figure 3}

Inter-hemispheric proteomic profile of human brain; Figure 3A illustrates the list of significant proteins which segregates Left hemisphere and Right hemisphere of Cerebral Cortex (Frontal Cortex, Occipital Cortex, Temporal and Parietal Cortex). Figure 3B depicts the list of proteins which segregates Left hemisphere and Right hemisphere of Basal Ganglia (Putamen, Lenticular nuclei and Caudate Nucleus). Figure $3 \mathrm{C}$ portrays the expression of few Cerebellar vermis enriched protein in terms of both the 
hemisphere and also the protein-protein interaction map of CBLN3, CBLN1, PCP2, NRXN1 and NRXN2. Figure $3 \mathrm{E}$ depicts the perturbation of interhemispheric regions of Brainstem, Pons and Substantia nigra. Figure 3F shows the protein-protein interaction of a biological process, Generation of Neurons (G0:0048699) with 18 Dentate Gyrus protein (17 Upregulated in Right hemisphere and 1 Downregulated in Left hemisphere).

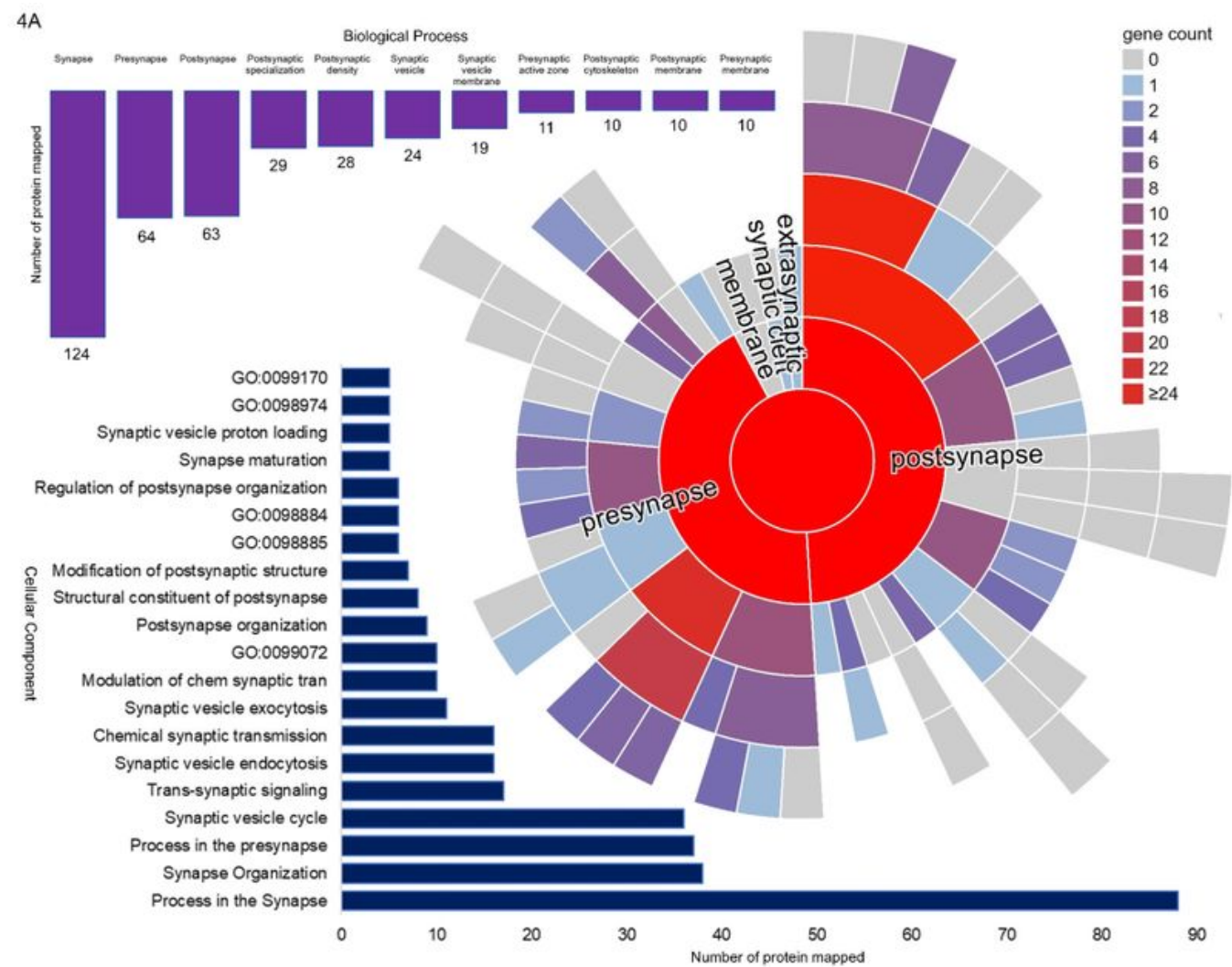

GO:0099072: Regulation of postsynaptic membrane neurotransmitter receptor levels; GO:0098885: Modification of postsynaptic actin cytoskeleton; GO:0098884: Postsynaptic neurotransmitter receptor internalization; GO:0098974: Postsynaptic actin cyloskeleton organization; GO:0099170: Postsynaptic modulation of chemical synaptic transmission Glutamatergic Synapse

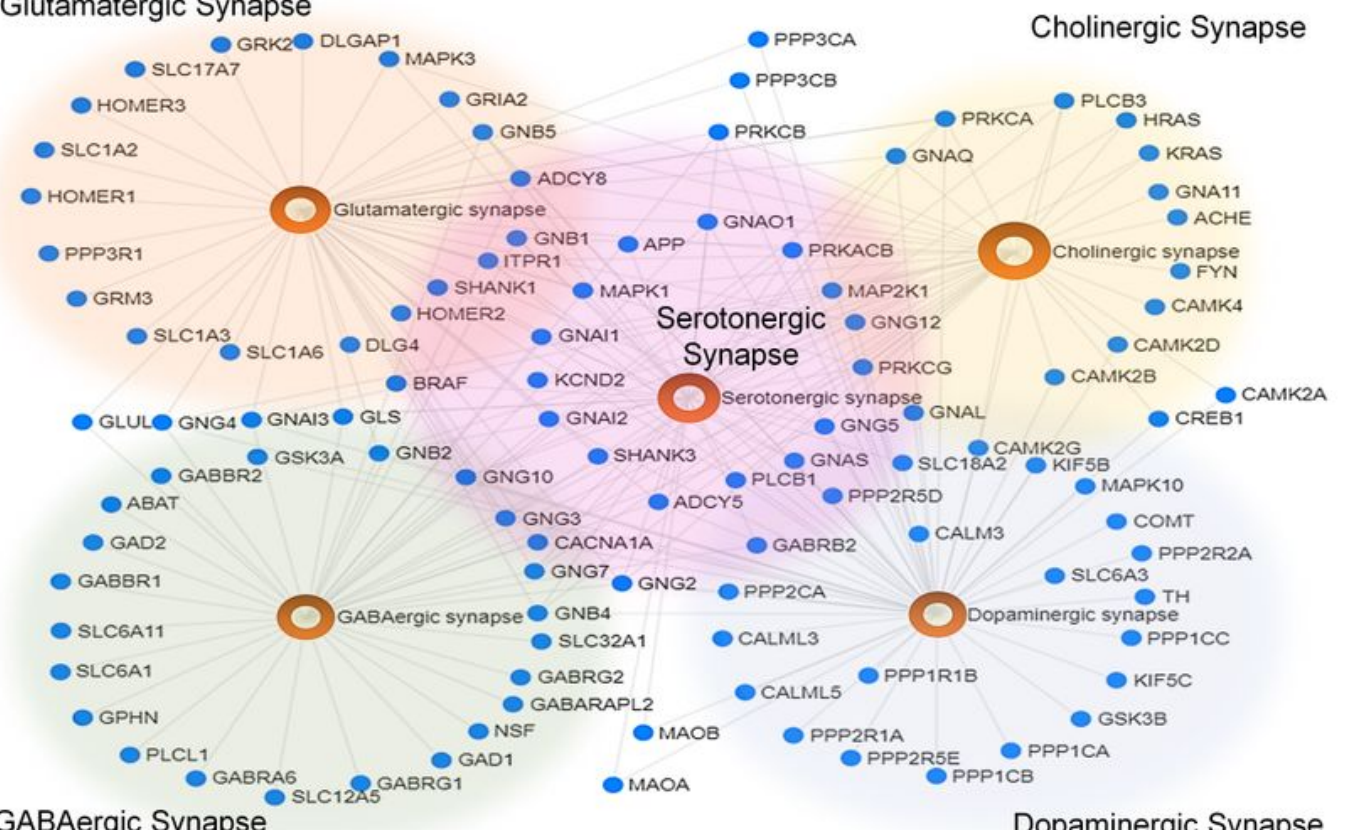

GABAergic Synapse

Dopaminergic Synapse

\section{Figure 4}


Biological analysis of Human Brain proteome; Figure 4A represent the synaptic gene ontologies in terms of biological processes and cellular component. Figure 4B depicts a gene bipartite network clusters which includes pathways like Glutamatergic Synapse, Cholinergic Synapse, Serotonergic Synapse, GABAergic Synapse and Dopaminergic Synapse.

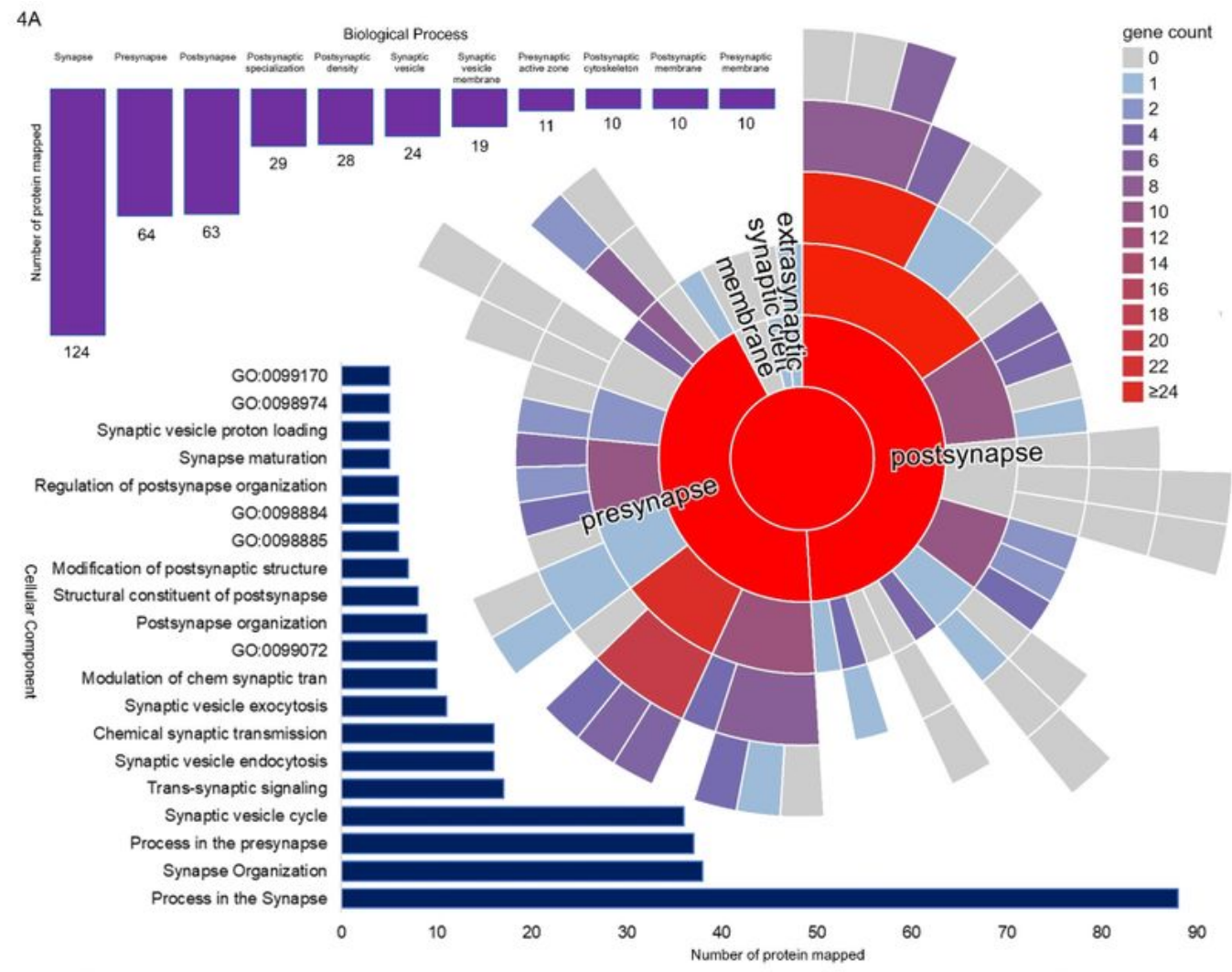

GO:0099072: Regulation of postsynaptic membrane neurotransmitter receptor levels; GO:0098885: Modification of postsynaptic actin cytoskeleton; GO:0098884: Postsynaptic neurotransmitter receptor internalization; GO:0098974: Postsynaptic actin cyloskeleton organization; GO:0099170: Postsynaptic modulation of chemical synaptic transmission Glutamatergic Synapse

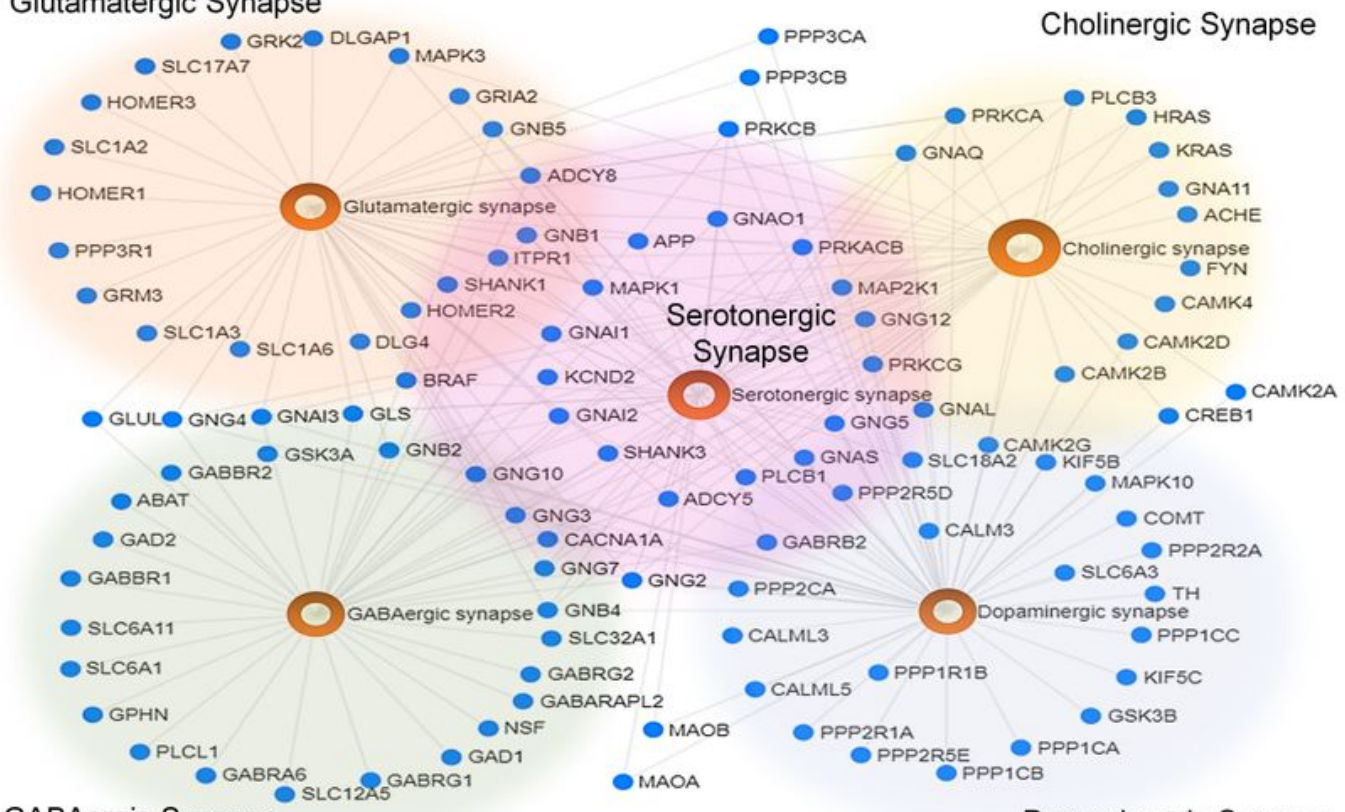

GABAergic Synapse

Dopaminergic Synapse

\section{Figure 4}


Biological analysis of Human Brain proteome; Figure 4A represent the synaptic gene ontologies in terms of biological processes and cellular component. Figure 4B depicts a gene bipartite network clusters which includes pathways like Glutamatergic Synapse, Cholinergic Synapse, Serotonergic Synapse, GABAergic Synapse and Dopaminergic Synapse.

\section{Supplementary Files}

This is a list of supplementary files associated with this preprint. Click to download.

- SupplementaryFigure1.jpg

- SupplementaryFigure1.jpg

- SupplementaryFigure2.jpg

- SupplementaryFigure2.jpg

- SupplementaryFigure3.jpg

- SupplementaryFigure3.jpg

- SupplementaryTableS1.xlsx

- SupplementaryTableS1.xlsx

- SupplementaryTableS2.xIsx

- SupplementaryTableS2.xIsx

- SupplementaryTableS3.xlsx

- SupplementaryTableS3.xIsx

- SupplementaryTableS4.xlsx

- SupplementaryTableS4.xlsx 